\title{
SYPHILITIC INOCULATION,
}

\author{
AND ITS \\ RELATION TO DIAGNOSIS AND TREATMENT.
}

\author{
BY \\ HENRY LEE, F.R.C.S., \\ ASSISTANT-SURGEON TO ST. GEORGE'S HOSPITAL, AND SENIOR SURGEON \\ TO THE LOCK HOSPITAL AND ASYLUM.
}

Received May 20th.-Read June 25 th, 1861.

Some of the points referred to in the following communication I have already had the opportunity of laying before this Society, and some others have previously been alluded to elsewhere, but the whole of the facts connected with syphilitic inoculation have not hitherto, so far as I am aware, been collected in a condensed and separate form.

The most ordinary kind of syphilitic inoculation is that in which the morbid action commences immediately upon the application of the poison, and produces a well-formed pustule within the first few days; but the form of disease which is of so much importance, as far as the patient's constitution is concerned, is one which often has a period of incubation, usually of some weeks' duration. It commences as a pimple, a crack, or an abrasion, and results in the adhesive form of inflammation. The first of these diseases is generally, but not always, developed upon a part which retains much of its natural softness or pliability; the second is generally, but not always, developed upon a part which becomes indurated in a very peculiar and characteristic manner. In cases where the induration fails to furnish the requisite information, the nature of the secretion will, as a rule, supply it. The first-mentioned morbid 
action will produce well-formed pus, which can ordinarily be recognised by the naked eye, and this product will continue puriform until the disease terminates; the second morbid action will, during a great part of its course, produce no pus at all, and in cases where for a short period pus is produced it will depend upon accidental cause, such as would produce suppuration from any abraded or irritable part. In other diseases the different kinds of inflammation which are produced as the result of inoculation have not been unnoticed. Thus, in describing the effects of the vaccine virus, Jenner ${ }^{1}$ remarks that pustulous sores often appear spontaneously on the nipples of cows; and that instances have occurred, though very rarely, of the hands of the servants employed in milking being affected with sores in consequence. These pustules, he observes, are of a much milder nature than the results of the contagion which constitutes the true cowpox. They are, he says, incapable of producing any specific effects upon the human constitution, and are noticed lest a want of discrimination should lead to the idea that the persons affected were in any measure thereby secured from the infection of the smallpox.

In a paper which I had the honour of laying before this Society in the year 1856, the corresponding differences in the kind of inflammation produced by the inoculation of different forms of syphilitic disease were insisted on, and the fact particularly pointed out that the secretion of the adhesive form of inflammation could often not be inoculated a second time upon the system which had produced it. These conclusions I have now reason to believe are generally acknowledged by the profession, although as late as the year 1858 we find : M. Ricord, in his 'Leçons sur le Chancre," ${ }^{2}$ telling his pupils, "Ce que je veins de dire de l'inoculation et des développements du chancre, se rapporte presque aussi bien a l'une qu'à l'autre de ses variétés." The period of incubation which so often occurs in the adhesive action, and the difficulty. with which that action

1 'Jenner on the Cowpox,' ed. 1800, p. 7.

2 Vol. x, p. 31 . 
can be reproduced, present several points of the greatest practical interest. These will be included in the present inquiry.

Besides the adhesive, and the suppurative actions, the application of the syphilitic poison is liable to be followed by two other distinct morbid processes. In one of these the virus is taken up by the lymphatics, and generally reproduces its specific action in the absorbent gland into which these first empty themselves; in the other, the part infected passes into a state of mortification, which may involve the destruction of the poison. Similar morbid actions have been observed after the application of other animal poisons to the surface of the human body. Thus, Jenner ${ }^{1}$ remarks that the variolous matter may undergo such a change from the putrefactive process, as well as from some of the more obscure and latent processes of nature, as will render it incapable of giving the smallpox in such a manner as to secure the human constitution from future infection. At the same time, he observes, the variolous matter so changed is capable of exciting a disease which bears so strong a resemblance to it as to produce inflammation and matter in the incised skin, swelling of the axillary glands, general indisposition, and eruptions. "This spurious action is often accompanied by more violent inflammation than that which occurs when the variolous matter produces its perfect effect upon the system." 2 These remarks are not intended to imply the existence of any strict analogy between the action of the syphilitic and other morbid poisons, but to show that any of these may be so modified by accidental circumstances and constitutional peculiarities as to produce different kinds of action under different circumstances.

The observations and illustrations which follow will have reference to the two principal kinds of syphilitic inoculation only, namely, that which produces the specific adhesive inflammation and that which is followed by the suppurative inflammation. The complications resulting from lymphatic

1 ' On Vaccine Inoculation,' ed. 1804, pp. 31, 32.

2 Ibid. 
absorption and mortification will, therefore, not be further noticed at present.

Whenever a sore which, if left to itself, will infect a patient's constitution, can be traced through its different stages, it will always, I believe, at some period or other, present the characters of the specific adhesive inflammation. The sores which, on the other hand, do not, in their natural course, infect a patient's system present the characters of the suppurative inflammation. The secretion produced by the adhesive inflammation consists of globules of different sizes and shapes, mixed commonly with epithelial débris, and floating in a transparent fluid. The globules may assume every appearance, from that of an ill-formed puscorpuscle to that of an epithelial scale. It will sometimes happen that the primary infecting sore produces no fluid secretion whatever, and then epithelial scales, more or less perfectly formed, can alone be obtained from its surface. It is true that these sores often do suppurate for a short time, or are preceded by other affections that yield a puriform secretion, but nevertheless the distinctive characters of the adhesive inflammation will always, I believe, be established before the occurrence of secondary symptoms.

The suppurating, non-infecting, sores, on the contrary, yield a purulent secretion during the whole time of their continuance. The difference between the secretion of an infecting and a non-infecting sore is shown in the accompanying illustrations, which were drawn on wood by my

Fig. 1.

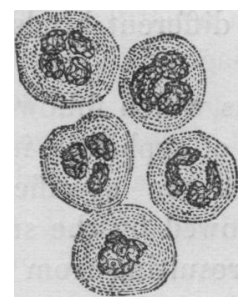

Microscopic appearance of secretion of local suppurating syphilitic sore, treated with acetic acid, $\times 700$.
Frg. 2.

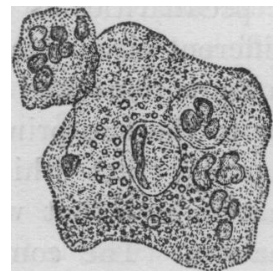

Microscopic appearance of cells of altered epithelium of an infecting sore, $\times 700$. 
friend, Professor Beale. Fig. 1 represents the secretion from a suppurating, non-infecting sore, when treated with acetic acid, and examined under the microscope with a power of 700 diameters. Fig. 2 represents the secretion from an indurated, infecting sore, treated in the same way and examined with the same power.

As a rule, the secretion from an infecting sore produces no result when inoculated upon another part of the same patient. Inoculation, then, in such cases, is worthless as a means of diagnosis, except in respect of the negative evidence which it affords; but although the secretion from an infecting sore cannot usually be inoculated upon the same patient, the habitual communication of the disease from one person to another shows that it may readily enough be inoculated on another individual who has not, previously had the same affection. In cases, however, where an infecting sore has been irritated, the secretion from its surface will become altered in its characters, and then it will, if inoculated upon the patient himself, produce an irritation or even a pustule of a few days' duration, but this will bear no resemblance to the original disease.

The drawing, Plate VIII, fig. 1, represents an indurated sore, the secretion of which, when inoculated, gave no result; but, after the sore had been made to suppurate artificially, the inoculation of its secretion produced a kind of pustule, which is represented on the eighth day of its appearance in Plate VIII, fig. 2; after this the abortive pustule began to die away, and is represented declining on the ninth day (fig. 3) ; it then soon disappeared altogether.

Inoculation of the secretion from a naturally suppurating sore furnishes an accurate mode of diagnosis. The effect commences upon the application of the poison, and results in a well-developed pustule within a week. This action constantly follows the application of the poison to an abraded surface, or its introduction beneath the cuticle, and it may be repeated in succession from the same original source a very great number of times. But, however often repeated, it does not produce constitutional syphilis, and therefore those 
who have practised this mode of treatment should not have named it "syphilization." The true syphilization is that disease which is communicated to a patient's constitution by the infecting sore, and there is too much reason to believe that this is not unfrequently accidentally conveyed from one person to another in the ordinary and normal relations of life.

It sometimes happens that the suppurating sore is surrounded by a considerable amount of induration, and that that induration, from some particular circumstances, terminates abruptly, very much in the same way as the induration around an infecting sore. The kind of secretion from the surface of the sore will then afford a means of diagnosis, if the case be uncomplicated and the part has not been subject to any accidental cause of irritation. But if a doubt should still remain, the inoculation of the secretion upon another part of the patient's body will, in unmixed cases, furnish the solution. If the sore be of the naturally suppurating kind, the specific pustule will result within a few days. If the sore be of the indurated and infecting class, the inoculation will be followed by no result, or at most such as has been already illustrated in Plate VIII, figs. 1, 2, 3.

Some months ago a boy presented himself at King's College Hospital. ${ }^{1}$ He was fourteen years old, and small for his age. There were no signs about him of his having arrived at puberty. He had had, as he said, one intercourse only. There was therefore the less chance of his having received more than one kind of infection. This lad, when he presented himself for advice had a phimosis, beneath which a circumscribed induration could be felt; a gland in the left groin was enlarged and hard. From the information derived from sensation alone $I$ had no doubt that this was an infecting sore, and, therefore, I sent him to the Lock Hospital, in order that he might have a proper

- The cases referred to in this paper are given at length in an Appendix. 
course of mercury. Before, however, beginning the treatment, I examined the secretion from the sore with the microscope. To my surprise, I found it distinctly purulent. The nature of the secretion then appeared to contradict the evidence derived from the sense of touch, and the test of inoculation was had recourse to. The specific pustule was produced, and the secretion from this again produced two other specific pustules, of exactly the same nature. It appeared, therefore, that if this were an unmixed case, the evidence given by the sense of touch had been fallacious, as it was clearly contradicted by the nature of the secretion and by the test of inoculation. The patient was therefore directed to discontinue the mercurial course which he had commenced two or three days previously, and was treated by steel pills and other non-specific remedies. The Plate VIII, figs. 4 and 5, are copied from a drawing made by Dr. Westmacott after the phimosis had subsided, and when the artificial inoculations were healed. At this period the secretion from one of the original sores on the penis was still purulent. The patient was kept in the Lock Hospital until he appeared quite free from any disease, and for a considerable period afterwards presented himself occasionally as an out-patient at King's College Hospital. During the time that he remained under observation he had no constitutional affection whatever, and I feel satisfied that had he had any up to the present time he would have again applied for relief.'

In this case, then, the evidence afforded by the nature of the secretion and by the test of inoculation corrected the erroneous impression that would have been and was conveyed by the sense of touch alone.

There are cases, however, in which neither the sense of touch, nor the nature of the secretion, nor the result of inoculation, will give positive information as to whether the patient will have secondary symptoms. These are the mixed cases, in

1 This patient again showed himself at King's College Hospital on the 31st of May (seven months after infection), without the least trace of constitutional disease. 
which a twofold inoculation has taken place. They occur, for the most part, in those who have never suffered from constitutional syphilis, for the obvious reason that, in those who have so suffered, inoculation of one kind can rarely and with difficulty be performed.

Twofold inoculation may occur either in the same or in different parts, at the same or at different times. When it occurs in the same part and at the same time, the results of the inoculation of the secretion from the suppurating sore will first develop themselves, and, subsequently, the results of the inoculation of the secretion from the infecting sore. This depends upon the different period of incubation which naturally belongs respectively to each kind of disease.

It is probable that the two actions resulting from the two kinds of inoculation cannot go on exactly in the same part at the same time, but they may be in such close proximity, or may follow each other so closely in point of time, that their respective effects (as regards the character of the secretion, \&c.) cannot be closely distinguished. The secretions from two different kinds of inoculation may then become mixed together, and if inoculated in that condition, a twofold result may follow. In that case the suppurating sore would first run its course, having all the appearances of a soft, non-infecting chancre, and afterwards (perhaps as this sore was healing up) the adhesive and infecting form of inflammation would appear. The cases in practice which have led to the greatest confusion are those in which the inoculation of the secretion from a suppurating sore has followed, after the lapse of three or four weeks, the inoculation from an infecting sore. We have, then, the results of two kinds of inflammation, and their respective products in close proximity. The suppurative inflammation does not, then, prevent the infection of the patient's constitution; the adhesive inflammation does not prevent the appearances of the "specific pustule." The means of diagnosis which would refer these mixed sores either to the infecting or to the suppurating class exclusively are therefore absent. 
Much confusion has arisen and many grievous mistakes have occurred from the assumption that every sore must necessarily, at any given time, present the characters exclusively either of the infecting or non-infecting variety. Nature is, as I believe, always true to herself, and if interrogated fairly, she will furnish a faithful answer. But this answer is given at her own time and in her own way. If the surgeon demands an immediate response the first time he sees a syphilitic sore, he may be disappointed; but if he will carefully watch the course of the disease, he may with confidence rely upon the information he receives. If a persistent form of suppuration be established after inoculation, natural or artificial, that disease is of the non-infecting kind, as far as the patient's constitution is concerned. If the adhesive form of inflammation be established, although after the lapse of some weeks, secondary symptoms will follow. If both kinds of inflammation be present, then the patient has received a twofold inoculation, and each disease will run its course, modified, perhaps, by, but in spite of, the other.

Out of 100 consecutive cases which I collected some years ago, of sores which at that time $I$ had diagnosed as of the suppurating kind, two of the patients presented themselves subsequently with secondary symptoms. Upon making further inquiry, I found that in both these cases the patients had exposed themselves to more than one source of infection. A good example of this kind of double contagion is afforded in Case No. 2 in the Appendix. The patient here had an induration which presented all the characters of the specific adhesive inflammation, and this had produced its natural result, namely, a secondary syphilitic eruption. But he had also a puriform discharge from beneath the prepuce, which, being inoculated upon another patient, produced the "specific pustule." Now, in this case a considerable period must have elapsed for the development of the infecting sore and its consequent secondary symptoms, and the very great probability is that the disease which produced the suppuration was received some weeks after that 
which produced the infecting sore. This case is now referred to, particularly as it was from it that the discharge was derived for one series of inoculations to be presently described.

Much uncertainty has been thrown over the conclusions derived from the results of syphilitic inoculations from the uncertainty (often much exaggerated by authors) of the degree in which different individual constitutions might modify the effects of the poison. Some have gone so far as to attribute the different results of inoculation altogether to the difference in the constitutions of the patients. A case has lately presented itself to me in the Lock Hospital, where, from peculiar circumstances, fully detailed (Case No. 3) in the Appendix, I had an opportunity of trying the effect of the inoculation of the secretions from different kinds of syphilitic sores, primary and secondary, upon the same patient. The different series of inoculations were therefore carried on under precisely the same circumstances, and some of them at exactly the same time. And the differences, which were marked in the strongest possible manner, were entirely due to the difference in the nature of the secretions inoculated. The order in which the inoculations were made will be fully given in the appendix. At present I purpose to state briefly the effect of each series of inoculations, independently of the rest, and of the precise date at which they were performed.

Some of the secretion from a well-developed primary indurated sore, of from two to three weeks' duration, and containing no pus-globules, was taken upon two little points of bone, such as are used for the purpose of ordinary vaccination. The cuticle having been pierced by a lancet, the points of bone, charged with the secretion from the primary infecting sore, were introduced beneath it, and allowed to remain several hours. A kind of abortive pustule was produced in each situation. The secretion from this was again inoculated on the third day, and produced a small spot or pimple. Beyond this no result could be produced by any further inoculation from this source. The appearances of the first 
inoculations on the twelfth day, and of those second in order on the tenth day, are shown in Plate IX, figs. 8 and 9, which are copied from drawings by Dr. Westmacott.

Some of the secretion was taken from a highly inflamed mucous tubercle, which accompanied and formed part of a set of secondary symptoms which had appeared for the first time in another patient. This secretion was inoculated in the same way with the ivory or bone points, and the results were very similar to those produced by the inoculation of the secretion of the primary indurated sore. A kind of pustule was produced by the first inoculations, the pustular character depending probably in part upon the irritation produced by the prolonged contact of the bony points. The secretion from these first inoculations was again inoculated in the ordinary way with the lancet in two places, and two small, livid spots or pimples alone were produced. As nuch fluid as could be obtained from these was again inoculated, and similar spots or pimples were produced. After this no inoculable secretion could be obtained from any of this series.

The drawings in Plate VIII, marked 6, 7, and 8, represent this series of inoculations on the twelfth, the eighth, and the fifth day respectively; and Nos. 9,10 , and 11 represent the same series on the twenty-fourth, twentieth, and the seventeenth day respectively. After this the appearances gradually faded away, and no further results have appeared from any of the foregoing inoculations.

Had these inoculations been performed on a patient who had never had constitutional syphilis, an indurated and infecting sore would, after a certain period of incubation, in all probability, have followed; and I take for granted that the reason why such modified results only were obtained was that the syphilitic poison had, on a former occasion, produced its full effect upon this patient's system. As it was, the secretion from the primary indurated sore produced an affection which could be again inoculated a second time only, and the secretion from the inflamed mucous tubercles produced an affection which could be reinoculated 
a third time only. After this the appearances of the inoculations in both cases gradually died away.

In strong contrast to these results are the effects produced by the inoculation of the secretion of a mixed (indurated and suppurating) sore, and from the patient's own serpiginous sores.

As the previously mentioned inoculations had shown that this patient was susceptible to only a very slight and modified effect from the secretion of a primary infecting sore, the results now described must have been from suppurating action superinduced upon the adhesive in the patient from whom the inoculated matter was taken. (See Case 2.)

Some secretion received on the point of a lancet was inoculated at two points in the ordinary way. On the third day an incipient pustule (Plate IX, fig. 4) surrounded by a red areola, had appeared in each situation. These were allowed to run their course, and became superficial suppurating sores, of the size of a fourpenny piece. Their appearance is represented on the twelfth day (Plate IX, fig. 5). Some pus from their surface, taken as soon as fully formed, was reinoculated, and produced exactly similar superficial suppurating sores (represented in Plate IX, fig. 6). The inoculation third in order is also represented on the third day (Plate IX, fig. 7); as compared with the first inoculation in this series, at the same period (Plate IX, fig. 4), it appears to be quite as fully developed, and to be surrounded by as distinct an areola. The inoculations from this source were carried on through seven series, an ordinary suppurating sore being produced without fail on each inoculated point.

In the last series of inoculations to be noticed, the secretion was from the patient's own very irritable and serpiginous sores. Upon each inoculated point a very well-developed and fully formed pustule appeared within the first few days. These pustules were depressed in their centres, and when they broke left exceedingly irritable ulcers. These ulcers were extremely painful, and surrounded a deep-red margin. A series of three sets of inoculations were performed, and the poison appeared quite as active 
and virulent at last as at first. Plate IX, fig. 1, represents the first inoculation which was made, on the sixty-second day of its progress ; Plate IX, fig. 2, the exceedingly well-developed pustules resulting from the inoculations second in order, on the eighth day of their existence; and Plate IX, fig. 3, the circular, irritable sores that were left after the inoculations third in order, on the twenty-first day of their progress.

Considering that this patient had had his serpiginous sores for a period of between five and six years, and that, in spreading from one part to another, fresh portions of skin were constantly being inoculated, it might have been supposed that something like an immunity to further inoculation would by that time have been acquired. It is true this patient was syphilized, in the proper sense of the word, and, from some previous infection of his system, was not susceptible to the effects of inoculation, either from a primary indurated, or from secondary sores, except in a very modified manner; but it is equally clear that the long and continuous course of self-inoculation to which he had been subject had not rendered him insusceptible of inoculation from either the ordinary suppurating sore or the more virulent poison secreted by his own ulcers. From the result of this case, and from a few others in which $I$ have tried it, I do not doubt that fresh inoculations from suppurating sores during the time of their development check the activity of other diseases of the skin; and this they do, I conceive, by a species of counter-irritation. Under this mode of treatment ulcerations of the skin will heal, and syphilitic eruptions will disappear, but it is because other sources of irritation have been established. The secretion from the suppurating syphilitic sores is a convenient mode of keeping up such a kind of counter-irritation, because, without any pain to the patient, it ensures a continued suppuration for a certain number of days. This suppuration is usually of a very limited extent, but it may be carried on in a great number of places at the same time, and its duration may be accurately regulated by the number of successive inoculations performed. 
The secretion from the unmixed indurated or infecting sore is of no use in this so-called syphilization. It is for some form of constitutional disease that this mode of treatment has been particularly recommended, and here the inoculation of the secretion from the indurated sore, as a rule, produces no result; on the other hand, the secretion from the suppurating form of chancre, although it may afford a valuable mode of maintaining counter-irritation on the skin, cannot, from its nature, be considered as a means of curing constitutional syphilis, inasmuch as it is itself a local disease, and does not produce any effect of a syphilitic nature upon a patient's system. When the secretion from a mixed (i.e. an indurated and suppurating) sore is inoculated upon a patient who has had constitutional syphilis, the result is, as a rule, the same as if the secretion of a suppurating sore only had been used; but if the same secretion were inoculated upon a patient who had not had constitutional disease, then a suppurating sore would be first produced, and, after a certain period of incubation, an induration would, in all probability, appear on the same spot, and would be followed by secondary symptoms.

This twofold inoculation naturally leads to the inquiry whether any other animal poisons may be inoculated together with the syphilitic. The nature of the subject forbids any direct experiments upon this point, as far as the infecting variety of syphilis is concerned, and clinical observations must therefore be relied upon. Such observations, with regard to ordinary vaccination, are not wanting, but nevertheless satisfactory conclusions have not hitherto been established. Mr. Simon, in the year 1857, collected together the opinions of 539 members of the medical profession in the United Kingdom and elsewhere, as to whether (among other things) a true Jennerian vesicle was ever a vehicle for syphilis, and whether such a poison could be unintentionally inoculated instead of the true vaccine lymph. The answers of the very great majority were decidedly in the negative upon both points; but, nevertheless, many gentlemen of experience recom- 
mended caution with regard to the patient from whom the vaccine lymph was taken, and in some instances medical men of experience expressed their convictions that the syphilitic poison might be so communicated. Thus, for instance, Mr. Ackerly, of Liverpool, writes, "I have no doubt that syphilis has been communicated from a diseased to a healthy child by means of vaccination."

Dr. Bamberger, of Würzburg, says, "I am indeed convinced that contagious disease, syphilis, for instance, ' is communicable with the lymph in vaccination; nay, such a case has even happened a short time ago in a town but a few miles from this place. After due inquiry into all circumstances of the case, the practitioner was found guilty by the Court of Justice, and condemned to prison for several months."

Mr. Barber, of Stamford, says, "It is very possible that a minute quantity of blood may become mixed with the lymph, and we know not how small a quantity of blood may suffice to convey a constitutional or other taint."

Mr. Complin says, "Syphilis, I consider, might be communicated."

Mr. Douglas, of Bradford, says, "Twenty-five years' observation and experience lead me to conclude that the greatest care ought to be taken."

Dr. Lever, of Guy's Hospital, says, "I have known syphilis communicated to a child by the hand of a supposed, but legally educated, medical practitioner."

Mr. Startin is of opinion that the true Jennerian vesicle in a subject suffering under constitutional or acquired syphilis, may be the means of transmitting this disease, "which," he says, "he has many times seen transferred from such a vesicle."

Dr. Whitehead, of Manchester, mentions that he has "seen several instances of the transference of the syphilitic taint through the medium of vaccination." He believes that the inoculation of matter taken from a syphilitic sore, in any of its stages, is capable of producing its characteristic phenomena in the inoculated.

VOL. XLIV. 
Now, it must be remembered, that these opinions were collected at a time when the doctrines of Ricord (as to the effects of syphilitic inoculation appearing, immediately after the application of the poison) were well nigh universally received. At that period if no results showed themselves at the expiration of the first week, none other were considered possible. The popular belief then prevailing among medical men is well expressed by the published answer of $M$. De Meric, who says, "I do not believe that secondary symptoms can be transmitted by inoculation; hence (if $\mathrm{I}$ am right in holding Hunter's ${ }^{1}$ and Ricord's opinion on this non-transmissibility) it is impossible to convey syphilis by vaccination, except the lancet have come in contact with pus secreted by a primary ulcer."

We have now been taught by clinical observation, and also by direct experiment, that the form of syphilis which infects a patient's constitution, has usually a period of incubation of some weeks before its effects develop themselves. And, inasmuch as it is this form of syphilis alone which is likely to be communicated by vaccination (and which alone would be of much consequence if it were so communicated), a fresh series of observations, aided by the additional light recently obtained, are required before the questions propounded can be considered as satisfactorily answered. Aided by recent investigations, Dr. Veinnois, in his thesis presented to the Faculty of Medicine in Paris in the year 1860, and also in the 'Archives of Medicine' for the same year, has collected together and given a detailed account of some cases in which an infecting syphilitic sore, or more properly speaking, the specific adhesive inflammation, followed vaccination. ${ }^{2}$ Dr. Veinnois has carefully excluded those cases in which a fresh set of symptoms followed vaccination in patients who had previously had hereditary or acquired syphilis, and has confined himself to cases in which the primary affections could be clearly

' Hunter said that the secretions from secondary syphilitic infection were not inoculable on the same body.

= An abstract of these cases will be given in the Appendix. 
verified, and their effects upon the constitutions of the patients satisfactorily traced. Dr. Veinnois' investigations have led him to the belief that, if the lymph from a vaccine vesicle be alone inoculated, the cow pox alone will be produced, but that if, in addition to this, the blood of a person affected with constitutional syphilis be inoculated at the same time, then that syphilis may also be communicated. The cow pox would then appear first, as having a shorter period of incubation; and after a time the syphilitic tubercle (or primary specific inflammation) would make its appearance upon the inoculated part, and would in due course be followed by secondary symptoms. The cases which Dr. Veinnois has collected, are related with so many circumstantial details, that if correctly reported, they cannot fail to establish the fact that the poison of syphilis and that of the cow pox may be communicated, and sometimes have been communicated, at the same time.

The principal conclusions to which I have been led in reference to the different points which $I$ have now had the honour to lay before this Society, are, that there are two forms of syphilitic disease, distinguished in their origin by the adhesive and suppurative kinds of inflammation. That one is followed by constitutional symptoms, and that the other is not.

That the adhesive form of inflammation may be distinguished from the suppurative by the nature of the secretion which it produces, and by the results of inoculation.

That both kinds of action may be communicated to the same individual at the same time, and that then the suppurative action will develop itself first, as having the shortest period of incubation, and subsequently the adhesive action will run its regular course.

That when these two actions are developed upon the same part, the affection which results has not the characters exclusively of the adhesive or of the suppurative inflammation. A mixed form of disease presents itself.

That the specific adhesive inflammation may be communicated to a person who has not previously had the disease, 
either directly by means of the discharge from a primary sore, or more indirectly through the secretions of a person affected with secondary symptoms.

That this latter mode of communication is not so common as the former, and appears to take place in general only when the part from whence the secretion is derived is in a state of increased or unhealthy action.

That under the circumstances last named, any open sore or abraded surface on a syphilitic patient may furnish an inoculable secretion.

That such open sore, or abraded surface, may be caused by mechanical irritation, by any secondary form of eruption, by a vaccine vesicle, or by a local suppurating syphilitic sore, in a person previously syphilitic.

That the blood of a syphilitic patient may communicate syphilis to a person previously unaffected with the disease.

That the cow pox and syphilis may be inoculated at one and the same time, and that when such a twofold inoculation does take place, the results are, in some respects, analogous to those which follow the inoculation at the same time of an infecting and a suppurating sore.

That the pure vaccine lymph, even from a syphilitic subject will not communicate syphilis.

\section{APPENDIX.}

CASE 1.-Alexander John C-, æet. 14, but younger in appearance, applied to me at King's College Hospital in November, 1860. About a fortnight before he had had one connexion only. Upon examination it was found that he had phimosis, and beneath the skin a circumscribed induration could be distinctly felt. One of the glands in the left groin was enlarged. The induration beneath the prepuce was so perfectly well defined, and afforded to the touch so very much the sensation usually relied upon as 
diagnostic of infecting sores, that I directed this patient to go as an in-patient to the Lock Hospital for the purpose of having a proper course of mercury. He was admitted on the 24th of November. When seen on the 27th, it appeared that there was more discharge than would usually be produced by an infecting sore, and this was therefore subjected to microscopical examination. It was found to consist entirely of well-formed pus. The patient was now inoculated with his own secretion on the thigh.

December 1st.-The inoculation had produced a welldeveloped pustule, surrounded by a red margin.

3rd.- The pustule had given way, and its secretion was inoculated in two places lower down.

8th.-Examination of the secretion of the original sore showed it to be distinctly purulent. The inoculations, second in order (of the 3rd instant), had produced two welldeveloped specific pustules.

He was directed to discontinue the mercury, which the house surgeon had ordered him a few days after his admission into the hospital. The three inoculations on the thigh produced three ordinary superficial suppurating sores, unaccompanied by any specific induration. These sores were healing on the 18th, and on the 24th they had skinned over. They were still, however, tender to the touch and red. The circumscribed induration could still be very distinctly felt beneath the prepuce.

January 15th.-- Phimosis continues; the discharge from beneath it still distinctly purulent.

24th.-Phimosis relieved : an ulcer producing a purulent discharge still exists on the anterior part of the glans penis, around the orifice of the urethra. See Plate VIII, fig. 4.

February 14th.-Was discharged from the Lock Hospital as cured, having had no specific treatment, except the mercury which was administered for a few days after his first admission.

This patient showed himself at King's College Hospital perfectly free from any constitutional disease on the 31 st of May following. 
Case 2.-John T-, æet. 15, was admitted into the Lock Hospital on the 5th of April, 1861. It was found, upon examination, that he had phimosis: a purulent discharge issued from beneath the prepuce, which concealed a dense and circumscribed induration about an inch in length. A secondary eruption covered his body. He stated that the phimosis commenced two months ago, but no distinct history could be obtained with regard to the infection of this patient's system. The purulent discharge was inoculated on another patient (see Case 3), and produced the specific pustule only.

Case 3.- Henry C-, æt. 32, first came under my care at the Lock Hospital on the 10th of April, 1856. At that time there were some cicatrices of old sores upon the penis; a small fissure existed on the margin of the prepuce, and there was a poisonous bubo (tested by inoculation) with foul surface and excavated edges in the right groin.

He stated that four months previously some sores formed beneath the prepuce, which he was unable to retract. He attended St. Bartholomew's Hospital, under the care of Mr. Paget, and was subjected to a course of mercury. When the sores were nearly well, about two months after their first appearance, a bubo formed and was opened. Its surface assumed a foul character, and it remained without any disposition to heal for another two months. He was then admitted into the Lock Hospital, and underwent a great variety of treatment. He again took mercury, and the surface of the bubo was repeatedly destroyed with different kinds of caustic. In January following the bubo still remained open, when he was discharged from the hospital for drunkenness.

He applied again and was readmitted on the 24th of January, 1861. The original sore in the groin had now healed, but from the marks it had left it evidently had extended at different times over both nates and some distance down both thighs. The patient stated that the ulceration had never completely healed; once or twice it 
had very nearly done so, but it broke out again and spread to some fresh part, and thus it had continued, healing in one place and spreading in another, ever since. During this interval he had been for seven months in Guy's Hospital under the care of Mr. Hilton. The sore was here again destroyed with nitric acid and with potassa fusa. He was ultimately, as he says, discharged as incurable from Guy's Hospital, and went to Camberwell workhouse. He remained in the workhouse for two months and was then sent to Margate, where he remained three months; and he returned again to the workhouse. On the 8th of October, 1857, he went a second time into Guy's Hospital, under the care of Mr. Cock, and at the expiration of ten weeks was discharged with two sores, of the size of a shilling, on the nates, one on either side of the anus. He now returned to his work, and continued sufficiently well to follow his employment until January, 1858, when the sores again became painful, and he was readmitted into the workhouse. Here he remained until January, 1859. By this time the sores had spread considerably on the inner side of the nates. He was again admitted into Guy's Hospital under Mr. Cock, treated as before, and again discharged all but well. He now returned to his occupation, and continued his work, that of a carpenter, for nine months. The sores again spread and involved the buttocks on both sides.

In May, 1860, he was admitted once more into the workhouse, where he remained until he was admitted the second time into the Lock Hospital. On his admission there were the cicatrices of old ulceration on the nates and upper and back part of both thighs, and some ulcers forming parts of circles were still open on the left side. These were extremely painful, and presented, on their surfaces, a number of small rounded eminences, some of them half covered by newly formed skin. The ulcerations having existed for a period of between five and six years, and the patient having had the advantage of being treated by different hospital surgeons, but without permanent benefit, I thought the case one in which (other remedies having failed) 
repeated inoculation might be tried. Having explained the plan to the patient, and having obtained his full concurrence, I commenced the inoculations on the 5 th of February. Some pus was taken from case No. 2, and inoculated on the right side of the patient's abdomen. On the 8th, specific pustules were produced and are represented in Plate IX, fig. 4, on the third day; and in Plate IX, fig. 5, on the twelfth day. The secretion from the artificial inoculations was reinoculated seven times in succession, and always produced the specific pustules. The appearances of the inoculations second and third in order are given in Plate IX, figs. 6 and 7. The inoculation eighth in order from this source did not succeed.

At the same time that the above series of inoculations was going on, a second series was commenced from the patient's own serpiginous sores.

On the 5th of February, some matter was taken from a sore on the right side of the nates and inoculated upon the patient's thigh. It produced an exceedingly well developed pustule, which broke and gradually assumed the appearance of a serpiginous sore. This is well represented in a drawing by Dr. Westmacott, copied in Plate IX, fig. 1 . On the fifty-fourth day of the existence of this artificial sore some secretion from its surface was reinoculated in two places and produced two very fine pustules, represented (Plate IX, fig. 2) on the eighth day of their existence. The inoculations third in order from this source ran exactly the same course, and are represented (Plate IX, fig. 3) on the twenty-first day of their existence. The virus had apparently lost none of its activity, and the sores produced by inoculation from this source were so very painful and irritable that no further inoculations from these sores were attempted.

On the 9th of March some strong nitric acid was applied to the original serpiginous sores, as it had frequently been before, The inoculations were allowed to run their course.

On the 14th, the ulcers touched with the acid had assumed a more healthy appearance.

On the 28th, some secretion from some highly inflamed 
mucous tubercles, forming part of a secondary eruption which had recently appeared upon another patient, was inoculated on the right side of Henry $\mathrm{C}$-'s chest. This made the third series of inoculations which were going on at the same time. In order to ensure the prolonged contact of the matter, some of the secretion was in this instance taken on some points of bone or ivory, and these were inserted beneath the cuticle and retained by means of plaster in their position.

The inoculations produced patches of circular inflammation, and in one of these a kind of pustule formed. The secretion from this was again inoculated on the 1st of April, and two abortive pustules were produced. The secretion from these was again inoculated, and produced only small circular patches of inflammation without any fluid secretion. The appearances of this series of the inoculations on the twelfth, eighth, and fifth days are represented in Plate VIII, figs. 6, 7, and 8, and in figs. 9, 10, and 11 , on the twenty-fourth, twentieth, and seventeenth days respectively. After the inoculations third in order no further results could be obtained from this source.

On the 9th of April the secretion from a well marked primary indurated sore, of between two and three weeks, duration, was inoculated in two places by means of the ivory points on this same patient.

On the 11th, two small abortive pustules were produced, but it was at the time supposed that the prolonged contact of the ivory points may have had some part in producing these pustules. The secretion from these artificial inoculations was again inoculated, and the result was only some small and inflamed points, yielding no fluid secretion. No further result could be obtained from this source. The appearances produced in this fourth series of inoculations are represented (Plate IX, figs. 8 and 9). The inoculations first and second in order are shown on the twelfth and tenth days respectively.

On the 23rd of April all the inoculations in the third 
and fourth series had dried up. The original serpiginous sores had now much improved in appearance.

On the 16th of May the original serpiginous sores were again freely touched with nitric acid. The inoculations from this source were still extremely irritable, and were allowed to run their course.

May 23rd.-An inoculation, the eighth in order of the second above-mentioned series, was made this day.

30th.-The inoculation of the 23rd had produced no effect. The superficial suppurating sores produced by this series were now healing.

June 6th.- The original serpiginous sores had now healed, except in one point which still appeared very irritable. The inoculations belonging to the second series had been repeated without result. Fresh inoculations from the sores of the second series yielding the largest amount of purulent secretion were repeated

13th.-The inoculations last made had succeeded as before, showing that the failures recorded on the 30th of May and 6 th of June depended upon some accidental cause.

20th.-The inoculations of the second series still succeeded. The original serpiginous sores were again destroyed by nitric acid.

27th.-No fresh inoculations had been made. The whole of the inoculations of the second series had dried up.

July 5th.-The inoculations from the patient's own serpiginous sores were nearly healed, having been touched with nitric acid. The sores themselves still remained unhealed in two places, and are of the size of a fourpenny and sixpenny piece respectively.

12th.-One of the original serpiginous sores is still unhealed, and of about the size of a threepenny piece.

August 31st.-The original sore is still unhealed, and is now of the size of a shilling, with irregular and abrupt edges. 
Abstract of cases collected by Dr. Veinnois in the 'Archives Générales de Médecine' for the year 1860 (tome xv, p. 657, et seq.), in which Syphilis appeared to be communicated by vaccination.

Mme. N. N- was delivered of a child in 1858. This child was evidently syphilitic, and communicated the disease to more than one wet nurse. The child was vaccinated, and the uncle and aunt were vaccinated from the child. After the vesicles in these were dried up, a hard, rough crust formed, which was surrounded by a reddish yellow areola.

The uncle was soon covered with scabs, and had affections of the bones and ulcerations. His symptoms were not cured until the expiration of five years. The aunt had ulcerations of the vulva and condylomata around the anus; she had also enlargement of the cervical glands and inflammation of the eye. She recovered about the same time as her husband.

In the 'Medicinishe Zeitung' of Berlin for the 3rd of April, 1850, the particulars are related of nineteen cases of persons between the ages of eleven and forty, who were infected in consequence of vaccination. Three or four weeks after the vaccination, ulcers-syphilitic in appearance-made their appearance on the vaccinated points, and these were followed in the majority of cases with secondary symptoms. The child from whom the lymph was taken was apparently healthy at the time, but subsequently had syphilitic roseola, and died a few days afterwards.

The vaccine vesicles in this child were not regularly developed. It was not until the tenth day that any lymph was produced that was considered fit for use.

On the 4th of May, P-, æt. 25, was revaccinated in accordance with the regimental regulations. The vaccine virus was taken from the arm of another soldier, who, three 
months previously, had bad an infecting chancre. Abortive pustules only were produced. One of these became inflamed at a later period, and gradually assumed all the characters of an indurated chancre. Its base was hard, and it was accompanied by indolent enlargement of axillary glands in the armpit of the same side. Constitutional syphilitic affections followed. ('Gazette des Hôpitaux,' Paris, Août, 1859.)

Another soldier, vaccinated shortly after from the same source, had a series of symptoms of exactly the same nature, which resulted in confirmed syphilis.

These two patients had never had any other syphilitic disease. They were the last of a number who were vaccinated, and the gentleman who performed the operation recollected afterwards that at the time he had exhausted his supply of vaccine lymph, and had taken up a little of the blood of the part upon his lancet.

A child, six years of age, previously in good health and with healthy parents, was vaccinated. An ulceration appeared at the vaccinated point, and was a long time before it healed. An eruption then appeared on the child's body. At the expiration of three years the child had coppercoloured blotches on its arms, and ulceration of the throat. ('New York Medical Times,' August 2nd, 1854.)

Cases No. 2, No. 14, No. 56, and No. 57, from the tables of Dr. Whitehead, of Manchester, are related at length by Dr. Veinnois, as instances of syphilis communicated by vaccination.

Three medical men inoculated themselves with the fluid from the varioloid pustules of a child born of a syphilitic. mother. The first had nothing. On the second, syphilitic ulcers were produced, which were followed by secondary symptoms. The third was seen by M. Heine, who relates the observation, with syphilitic psoriasis, some years afterwards. ('Beiträge zur Lehre von der Syphilis,' Würtzburg, 1854.) 
To the above may, perhaps, be added a case which fell under my own observation at King's College Hospital.

J. W. T-, was vaccinated at the age of seven months, on the 16th of June, 1859, having been perfectly healthy up to that period. His mother appeared a perfectly healthy woman, and stated that her husband had always enjoyed good health. This little patient when brought to the hospital in the beginning of the year 1861 (having previously been subject to a variety of treatment) presented numerous dark copper-coloured patches over his body, and especially on his lower extremities. These I considered to be syphilitic. On examining the child's arm the cicatrix left by the vaccination was very apparent, and was slightly harder than the surrounding skin; but that which particularly attracted attention was the existence of chronic enlargement of a gland in the axilla. This was hard, accurately defined, and not tender to the touch. The child was treated by mercury, and recovered. The mother continued to bring him to the hospital long after he had lost his eruption. She also brought the medical certificate of successful vaccination under the compulsory vaccination act. 


\section{DESCRIPTION OF PLATE VIII.}

Fig. 1.-Irritated primary indurated sore.

" 2.-Inoculation from 1 , on the the patient's thigh, eighth day.

" 3.-The same inoculation, ninth day.

" 4.-Cicatrized primary sores on glans and prepuce, which caused phimosis, and were accompanied by considerable induration. This could not be distinguished from the induration of infecting sores. There was also enlargement of one gland in the left groin.

„5.-Inoculation from 4, on the patient's left thigh.

" 6.-Inoculations of secretion from secondary inflamed mucous tubercles on twelfth day.

„7.-Inoculations from inoculations (6), eighth day. These inoculations formed pustules, which had now dried up.

, 8.- Inoculations from inoculations (7), fifth day.

"9.-Inoculations from inflamed secondary mucous tubercles; appearances on the twenty-fourth day.-The same as 6 .

"10.-Inoculations second in order from 9; appearances on the twentieth day.-The same as 7.

"11.-Inoculations; third in order from 10; appearance on the seventeenth day.-The same as 8 .

\section{DESCRIPTION OF PLATE IX.}

Fig. 1.-Artificial inoculation on the sixty-second day, from a serpiginous sore of six years' duration.

,2.-Inoculations from inoculation (1) on the eighth day.

"3.-Inoculation, third in order, from patient's own serpiginous sore; appearance on twenty-first day.

"4.-Inoculations from indurated and infecting, but inflamed and suppurating sore.-Third day.

"5.-Inoculations from indurated, but suppurating sore ; appearance on twelfth day.

, 6.- - Inoculations, second in order from 5.

, 7.-Inoculation, third in order from 6, third day.

" 8.-Inoculation of secretion of primary indurated sore. No pusglobules in the secretion.-Twelfth day.

"9.-Inoculation, second in order from 9, tenth day. 

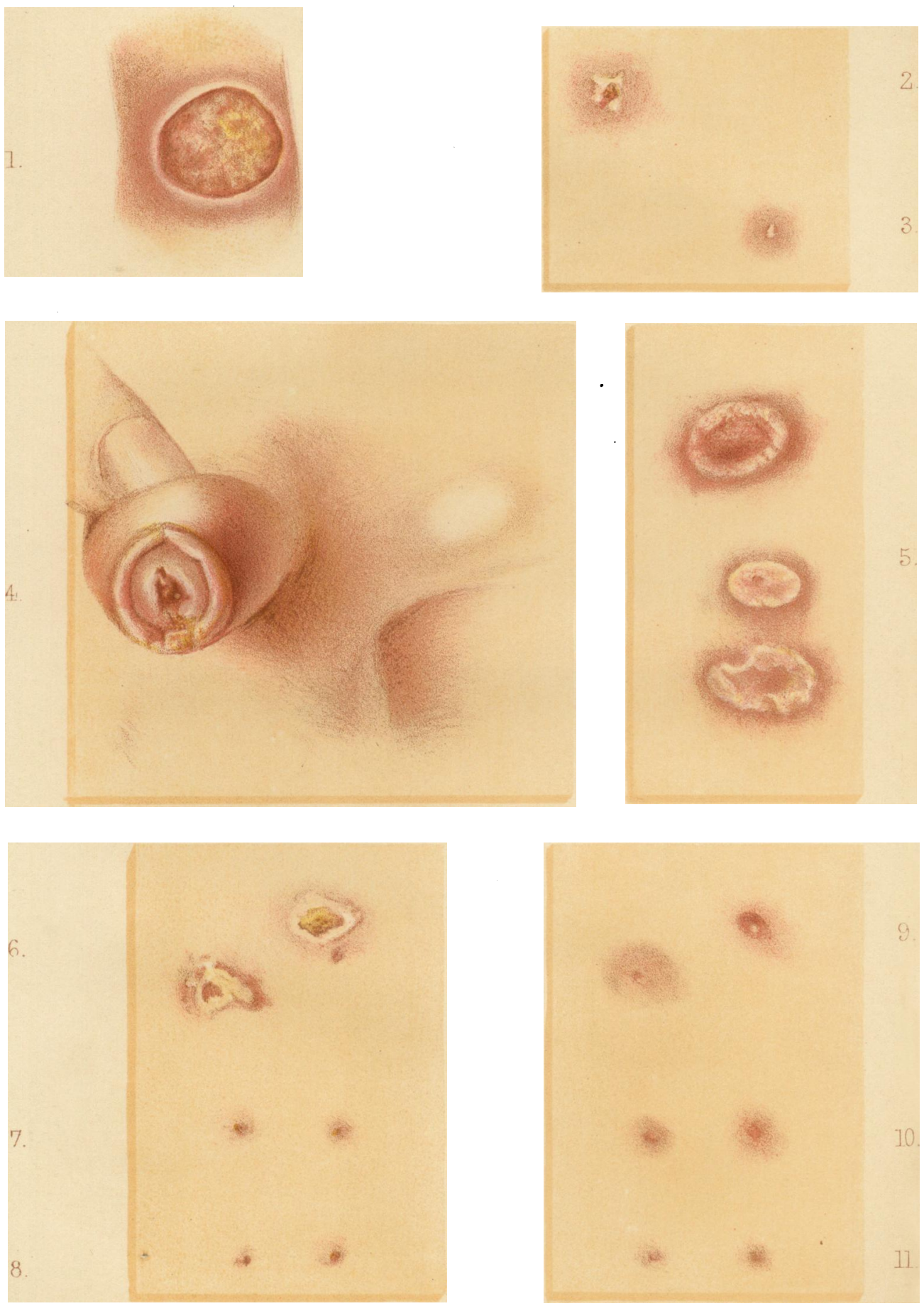
Plate IX.

Vol. XI,IV.

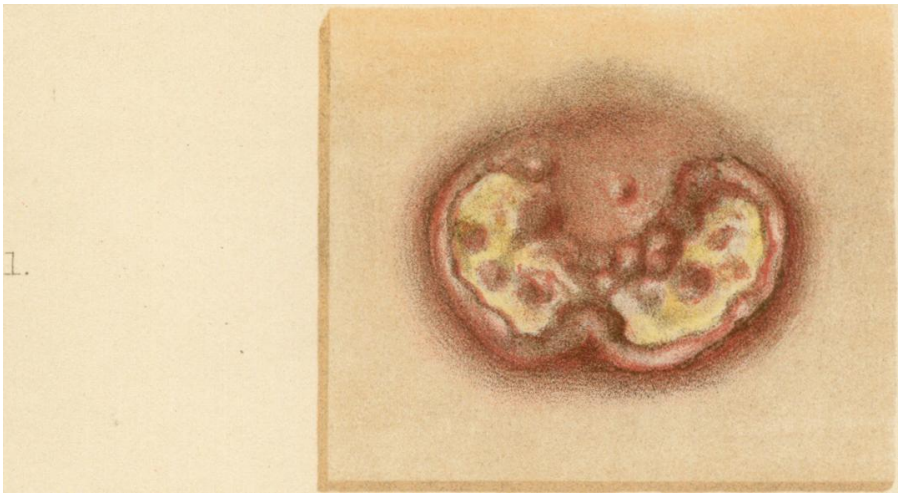

.
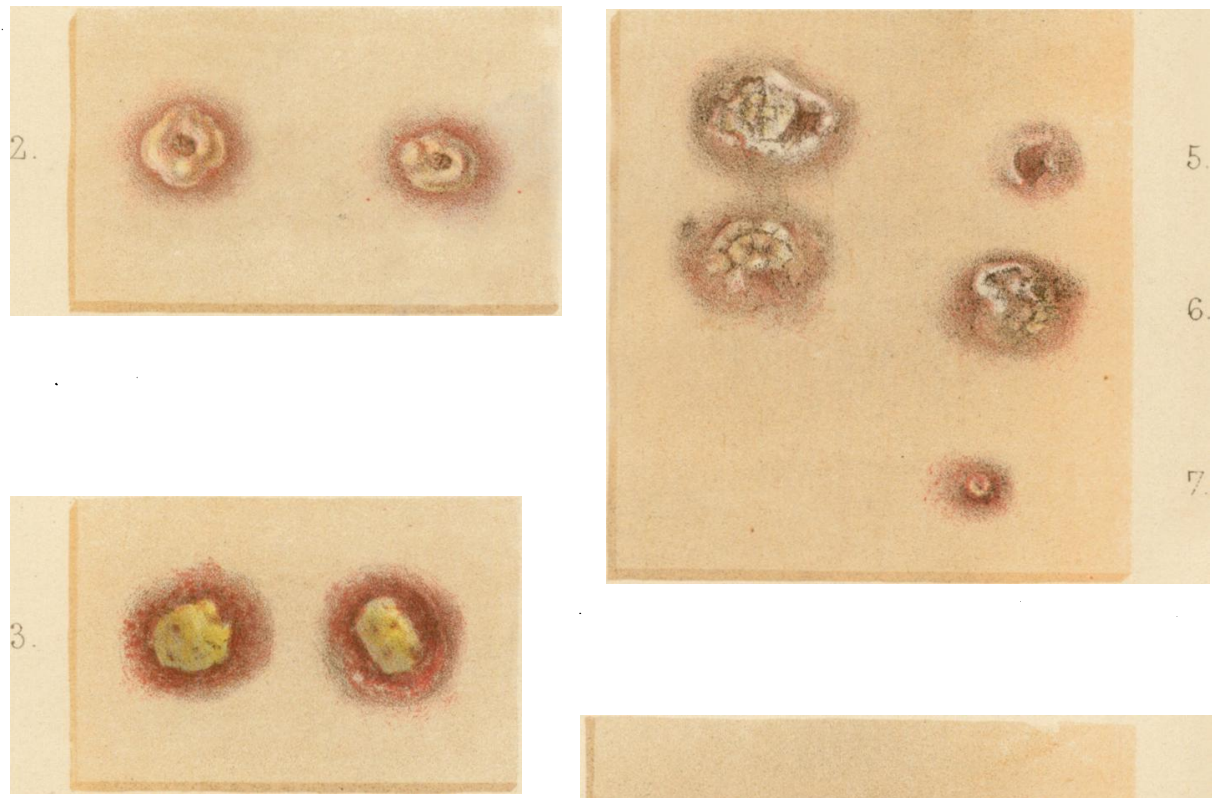

a
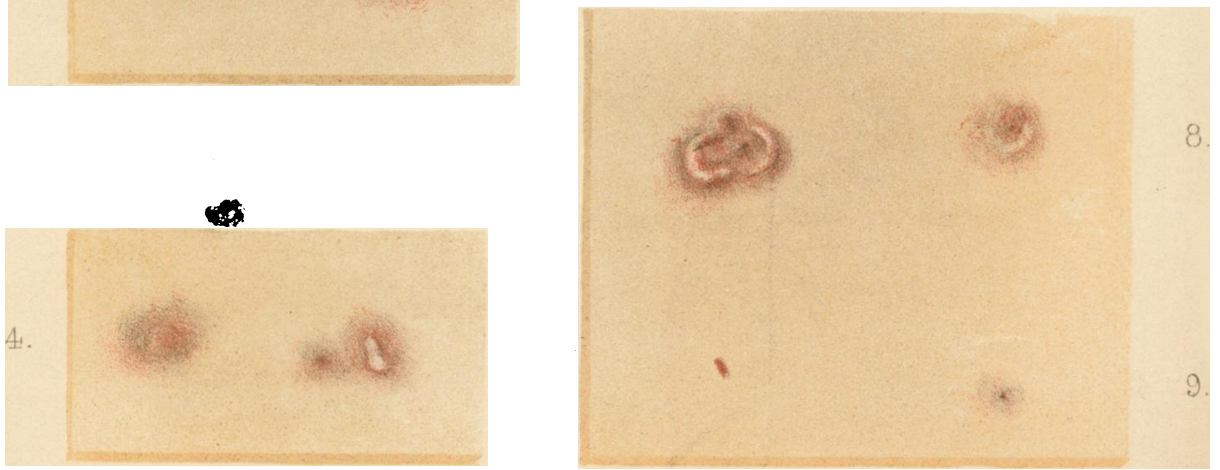